\title{
Extemporaneous Compounding: Cautions, Controversies and Convenience
}

\author{
AK Mohiuddin ${ }^{\star} \dagger$ \\ Assistant Professor, Department of Pharmacy, World University of Bangladesh, Bangladesh
}

DOI: https://doi.org/10.15520/ijmhs.v9i1.2420

Accepted 25 Jan 2018; Received 30 Dec 2018; Publish Online 30 Jan 2019

\author{
Reviewed By: Dr. \\ Daniel V. \\ Department: Medical
}

\begin{abstract}
Pharmacists are only knowledgeable and skilled healthcare professionals dedicated to compounding and preparing medications to meet the unique needs of patients. The safe and effective extemporaneous compounding of prescription products for patients require in special care is fundamental to the pharmacy profession. But there are much to do for secundum artem. It is not at all economical for a pharmaceutical company to marketize a product in 10 different probable doses or in 5 different dosage forms to meet the needs of the entire range of individuals receiving therapy. Although development is a continuous process, companies are customizing features to meet the majority of patient needs, but the very nature of the process cannot meet all patient needs. The risk-benefit ratio of using traditionally compounded medicines is favorable for patients who require specialized medications that are not commercially available, as they would otherwise not have access to suitable treatment. However, if an FDA-approved drug is commercially available, the use of an unapproved compounded drug confers additional risk with no commensurate benefit. Published reports of independent testing by the FDA, state agencies, and others consistently show that compounded drugs fail to meet specifications at a considerably higher rate than FDA-approved drugs. Compounded sterile preparations pose the additional risk of microbial contamination to patients. In the last 11 years, three separate meningitis outbreaks have been traced to purportedly 'sterile' steroid injections contaminated with fungus or bacteria, which were made by compounding pharmacies. The 2012 outbreak has resulted in intense scrutiny of pharmacy compounding practices and increased recognition of the need to ensure that compounding is limited to appropriate circumstances.
\end{abstract}

Purpose of The Study: The article aims to physico-chemical and economic considerations before compounding; factors and quality control issues; compounding support, training, chemical supplies, types of compounding (specially in hospital and ambulatory care compounding). It should aid to practice the extemporaneous preparation of basic and advanced formulations including pharmacopoeial and non-pharmacopoeial formulations encountered in pharmacy practice, together with requisite documentation, labeling, packaging and counseling requirements. Along with this, they have to study the analysis of formulations and their components and relate these to the clinical performance of medicines. This will help them to investigate, evaluate and report the physical characteristics of formulations including release kinetics and relate these to quality control and preformulation requirements; relate the application of quality control, quality assurance and the principles of good manufacturing practice to regulation of medicine production in home and abroad.

Outline: Background; Introduction; Compounding Factors; Types of Compounding; Identifying Errors and Cause

Key words: Pharmacy Practice; Drugs; Dosage Forms; Compounding; Equipment; Pharmacopeia 


\section{INTRODUCTION}

The FDA defines traditional pharmacy compounding as the combining, mixing, or altering of ingredients to create a customized medication for an individual patient in response to a licensed practitioner's prescription. The NABP further describes compounding as the result of a practitioner's prescription drug order based on the practitioner/patient/pharmacist relationship in the course of professional practice. Traditional pharmacy compounding plays a valuable role in providing access to medications for individuals with unique medical needs, which cannot be met with a commercially available product. For instance, a prescriber may request that a pharmacist compound a suspension for a pediatric or geriatric patient unable to swallow a medication in its commercially available form. In traditional pharmacy compounding, an individualized medicine is prepared at the request of a prescriber on a small scale. It is obvious that large scale manufacturers have very limited opportunity to customize dosage strength and dosage forms for a customer segment that is not substantially big enough due to economies of scale concept of business. Another major difference is that compounding pharmacies are not required to report adverse events to the FDA, whereas adverse event reporting is mandatory for manufacturers of FDA-regulated medications. Thus, adverse events associated with compounded drugs may be difficult to detect, particularly if the affected patients are widely scattered in different geographic areas. Registered and skilled pharmacists in practicing their art of compounding fills in this gap to meet individualized needs. Compounding has always been a basic part of pharmacy practice; the drugs, dosage forms, and equipment or techniques used are the variables. Pharmacists have unique knowledge and skills and are not duplicated by any other profession. Pharmacy activities to individualize patient therapy include compounding and clinical functions. Either function in the absence of the other results in placing pharmacy in a vulnerable position. It is important to entrust a pharmacist's expertise to adjust dosage quantities, frequencies, and even dosage forms to enhance compliance. Pharmacy is a complex mixture of different practices and practice sites. No longer is pharmacy simply community pharmacy or hospital pharmacy. Pharmacy is diverse and offers many opportunities for those willing to look around, find their niche and practice pharmacy to meet the needs of their own community of patients. Most compounding pharmacists appear to be interested and excited about their practices. In fact, many pharmacists intimately involved in pharmaceutical care have now realized the importance of providing individualized patient care through the preparation of patient-specific products. Compounding pharmacy is not for everyone, but as it grows, it will provide an increasingly significant number of pharmacists the excitement and fulfillment of using their innovative and creative skills to solve patient problems.

* Corresponding author.

$\dagger$ Email: trymohi(at)gmail.com

\section{Rationale of Extemporaneous Compounding}

An estimate by FDA that $1-10 \%$ of all prescriptions require compounding was not supported by evidence data. EP strategies in clinical studies requires 'fit-for-purpose' formulation design and preparation processes, as well as administration procedures that are safe, flexible, cost-effective, and simple to adapt by a compounding pharmacist at the clinical site. Many commercially available medications are not manufactured in suitable dosage forms for these special populations, e.g., liquid formulations for children and those who may be unable to swallow tablets. Additionally, when drug shortages occur or for the treatment of rare diseases, compounded products have a place in practice. Examples of altered drug products include topical ointments, eye drops, solid or liquid oral dosage forms, and intravenous infusions. Compounding also has a place in veterinary practice, where medicines may be altered to suit drug absorption, metabolism, and swallowing abilities of different species. The use of small-scale compounded medications carries certain risks, as they are not assessed for product quality, stability, or efficacy. The importance of a collaborative, team-based approach to patient care and safety has been well established within the health field. The requirement for alternative medications and dosage forms may be determined by the patient's general practitioner who prescribes the medication or other allied health professionals: a speech pathologist who identifies a swallowing difficulty, a physiotherapist applying ultrasound, which may interfere with transdermal drug delivery, or an occupational therapist identifying that a patient is unable to physically manipulate their current medication or its packaging. This highlights the importance for other members of the health care teams to have knowledge and understanding of compounded medications. Among elderly patients, the incidence of dysphagia is reportedly as high as $7 \%-13 \%$, making utilization of available oral dosage formulations unfeasible or unsafe. Similarly, Canadian hospital pharmacists often encounter patients with oral, neck, or gastric cancer, which may make commercial solid dosage formulations impossible to use. Based on pharmacists' perspective, using more extemporaneous prepared products could also make sense economically. 'The significant therapeutic improvement and some cost savings may be gained' by the rational use of extemporaneously prepared medicines. Pharmacists that did not provide compounding service cited by the main reason as not receiving prescriptions that required compounding (63.6\% of non-compounder pharmacists). In about $33 \%$ of the compounded products, they indicated patient-specific reasons. In about $10 \%$ this reason concerned a strictly defined pharmaceutical care issues. Some researchers conclusively said that $73.2 \%$ pharmacists reported their goal in providing full pharmaceutical care to their patients was the most important motivator. The main reasons for the decision to provide compounding service were want to provide full pharmaceutical care to patients (73.8\% of compounder pharmacist) and responding to requests by prescribers. Most community pharmacists (nearly $70 \%$ ) accepted compounding as a component of pharmaceutical care. In most of the studies, major reasons stated by 
physicians for prescribing extemporaneous products were that they worked well for certain conditions and that the product was not available commercially [1-7] .

\section{Evaluation of the Need}

Soluble or dispersible tablets may be a useful and convenient alternative to the preparation of liquid extemporaneous products. Some tablets can be dispersed or crushed and information on this aspect can be obtained from the Medicines Information Section within the healthcare setting. In this case, the dose should be prepared and administered immediately. In general, compressed tablets or tablets which are scored or just film coated can be crushed whereas modified release tablets cannot.

If a particular medicine is not available as a liquid formulation, another medicine from the same therapeutic classification may well be used, such as the use of a less potent steroid rather than diluting a potent one.

Using a suitable preparation intended for a different route of administration, for example, using an injectable solution orally.

Use of a 'specials' preparation manufactured in licensed premises (Specials are medicines made in larger volumes by a licensed manufacturer) [7] .

Considerations before compounding

Commercially availability of drug in dosage form, strength, and packaging

Ingredients, intended use, dosage, and method of administration concern

Education, skill and expertise to drug compounding

Proper equipment, supplies, chemicals and the guidelines delineated in us pharmacopeia

An alternative by which the patient will receive a benefit

Safety of the compounded product

Patient necessary storage facility, if required,

Necessary calculations to prepare the product

Necessary documentation to complete preparation

Literature reference that might provide information on use, preparation, stability, administration

Expected duration of therapy

Some basic quality control to check the product prior to dispensing (e.g., capsule weight variation, $\mathrm{pH}$, visual observations)

Ingredient identity, quality, and purity

Corrective methods

Physico-chemical incompatibilities [5, 6]

Economic Considerations: The most significant factor which limits prescribing of compounded medicines was that patients did not want products due to these products were not covered by the national insurance. Furthermore, for doctors not prescribing these products, the major barrier to prescribing was their lack of knowledge of the products. Most responding doctors do not prescribe medications that require compounding because they lack trust in the quality of the compounded formulations. However, most of the doctors expressed a desire to learn more as shown in having access to information about the usefulness of extemporaneous products. There are at least two different economic considerations in making the decision to compound prescriptions; these include (a) pharmacist compensation and (b) healthcare costs. The pricing of a compounded prescription should include consideration for pharmacodynamic and pharmacotherapeutic decision making, formulation expertise, time, and reimbursement of materials. Compounding prescriptions can be attractive professionally and financially. Compounding prescriptions can be a way of lowering the cost of drug therapy. In some cases, it is less expensive for the pharmacist to prepare a specific prescription for the patient, which may mean the difference between the patient actually obtaining the drug or doing without it. If compounding a prescription results in a patient being able to afford the drug therapy, it must be considered. An interesting example involves the drug 4-aminopyridine. Although physicians had been prescribing unapproved versions of the drug for up to 20 years, it was not until the drug was studied systematically that rare seizures were discovered as a potential side effect. In this case, the medical profession pushed for an approved version to be marketed, rationalizing that if a seizure occurred in the context of a patient taking an FDAapproved alternative $[4-10]$.

\section{COMPOUNDING FACTORS}

Stability: One key factor in compounding prescriptions is stability. The more common types of stability of which compounding pharmacists should be aware include chemical, physical, and microbiological. Whereas commercially manufactured products are required to possess an expiration date, compounded products are assigned a beyond-use date. There are numerous sources of information that can be used for determining an appropriate beyond-use date, such as chemical companies, manufacturers literature, laboratory data, journals, and published books on the subject. Generally, most pharmacists prepare or dispense small quantities of compounded products; recommend storage at room, cool, or cold temperatures; and use a conservative beyonduse date. For nonaqueous liquids and solid formulations (for which the manufactured drug product is the source of active ingredient) - The beyond-use date is not later than $25 \%$ of the time remaining until the product's expiration date or 6 months, whichever is earlier. A USP or NF substance is the source of active ingredient- The beyond-use date is not later than 6 months. For water-containing formulations (prepared from ingredients in solid form) - The beyond-use date is not later than 14 days when stored at cold temperatures. For all other formulations - The beyond-use date is not later than the intended duration of therapy or 30 days, whichever is earlier. These beyond-use date limits may be exceeded when there is supporting valid scientific stability information that is directly applicable to the specific preparation (i.e., the same drug concentration range, $\mathrm{pH}$, excipients, vehicle, water content). For example, active ingredients containing an ester functional group, such as aspirin and penicillins, are susceptible to breakdown by hydrolysis, while those containing aldehyde or hydroxyl groups, such as testosterone and dopamine, undergo oxidative decomposition. A $10^{\circ} \mathrm{C}$ increase in temperature can result in a 
2-5-fold faster rate of degradation. The instability of dapsone suspensions prepared in Oral Mix and stored at $25^{\circ} \mathrm{C}$ may be explained by a Maillard reaction between the sucrose in the vehicle and the amino groups of the dapsone. The results of this study have demonstrated the stability, for up to 90 days, of dapsone suspensions $(2 \mathrm{mg} / \mathrm{mL})$ prepared from commercial tablets in Oral Mix SF and stored at $5^{\circ} \mathrm{C}$ and $25^{\circ} \mathrm{C}$ or prepared in Oral Mix and stored at $5^{\circ} \mathrm{C}$, in amber plastic bottles and amber plastic syringes. These suspensions should be shaken before use $[2,5,6,11,12]$.

It is essential that the active ingredient does not interact with any excipients originating from the dosage form being crushed for reformulation (e.g. tablet lubricants and fillers), or from additives in the new formulation (antioxidants, preservatives, suspending agents, colourants, emulsifiers). Interactions with other ingredients can result in physical instability of the product, such as precipitation of the active drug or phase separation ('cracking') of a cream, affecting drug solubility, absorption and bioavailability. To minimize the risk of a compounded medicine degrading, short-term expiry dates are used (e.g. 28 days for oral and topical products, or 24 hours for parenteral formulations), unless stability studies have been conducted and indicate otherwise $[2,5]$.

Potential Risks: FDA-approved drugs are made and tested in accordance with GMPs, which are federal statutes that govern the production and testing of pharmaceutical products. In contrast, compounded drugs are exempt from GMPs, and testing to assess product quality is inconsistent. Unlike FDA-approved drugs, pharmacy-compounded products are not clinically evaluated for safety or efficacy. The NABP further describes compounding as the result of a practitioner's prescription drug order based on the practitioner/patient/pharmacist relationship in the course of professional practice. In addition, compounded preparations do not have standard product labeling or prescribing information with instructions for safe use. Compounding pharmacies are not required to report adverse events to the FDA, which is mandatory for manufacturers of FDAregulated medications. Some pharmacies engage in activities that extend beyond the boundaries of traditional pharmacy compounding, such as large-scale production of compounded medications without individual patient prescriptions, compounding drugs that have not been approved for use in the US, and creating copies of FDA-approved drugs. Compounding drugs in the absence of GMPs increases the potential for preparation errors. When compounding is performed on a large scale, such errors may adversely affect many patients. Published reports of independent testing by the FDA, state agencies, and others consistently show that compounded drugs fail to meet specifications at a considerably higher rate than FDA-approved drugs. Compounded sterile preparations pose the additional risk of microbial contamination to patients. In the last 11 years, three separate meningitis outbreaks have been traced to purportedly 'sterile' steroid injections contaminated with fungus or bacteria, which were made by compounding pharmacies. US-FDA and the NICHD, have worked together to develop product labeling for pediatric patients for existing and new drug molecules coming to the market, if the drug has been or could be used in children. When compared to GMPs, the manufacturing standards required by FDA for commercial products, there are multiple recognized deficiencies inherent in extemporaneous formulation-compounding suitable for pediatric patients $[11,13]$

Quality Control: One of the fastest growing and most important areas of pharmaceutical compounding is that of quality control. Quality must be built-in to the preparation from the beginning steps to evaluating the final preparation. The extent of quality control applied to a product should be proportionate with the level of risk the finished product could pose to the patient and should be judged on a case by case basis. Even where a given formulation has been shown to achieve suitable physical, chemical and microbiological stability, the bioavailability and palatability of the preparation may be unproven. Consideration should be given to the individual patient's age and condition, whether the product will be ingested or used topically, as well as the potency of, and risk posed by, the active substances and/or excipients. Where it is not practical to carry out this testing (e.g. due to the batch size, urgency, etc.), other suitable methods should be implemented to ensure that the appropriate quality is achieved, in accordance with the risk assessment carried out. At a minimum, the starting materials and finished product should be examined visually before supply to a patient. There are several quality control tests that can be done within the pharmacy and others can be sent to a contract laboratory. The following quality control tests can be considered for the respective dosage forms.

Oral and topical liquids (solutions, suspensions, emulsions): Weight/volume, $\mathrm{pH}$, specific gravity, active drug assay, globule size range, rheological properties/pourability, physical observation (color, clarity), physical stability (discoloration, foreign materials, gas formation, mold growth).

Hard Gelatin Capsules: Weight-overall average weight, weight individual weight variation, dissolution of capsule shell, disintegration and/or dissolution of capsule contents, active-drug assay, physical appearance (color, uniformity, extent of fill, locked), physical stability (discoloration, changes in appearance).

Ointments, Creams and Gels: Theoretical weight compared to actual weight, $\mathrm{pH}$, specific gravity, active drug assay, physical observations (color, clarity, texture-surface, texture-spatula spread, appearance, feel) and rheological properties.

Suppositories, Troches, Lollipops and Sticks: Weight, specific gravity, active drug assay, physical observation (color, clarity, texture of surface, appearance, feel), melting test, dissolution test, physical stability.

Parenteral preparations: Weight/volume, physical observation, $\mathrm{pH}$, specific gravity, osmolality, assay, color, clarity, particulate matter, sterility, pyrogenicity [5, 6, 14] .

Compounding Support: Numerous agencies, companies, organizations, etc., are available to assist pharmacists in compounding. Information, chemicals, supplies, and 


\begin{tabular}{|c|c|c|c|}
\hline \multicolumn{4}{|c|}{ Exhibit 1 . Common degradation pathways of active drugs in compounded products* $[2,5]$} \\
\hline Pathway & $\begin{array}{l}\text { Factors determining } \\
\text { degradation rate }\end{array}$ & $\begin{array}{l}\text { Susceptible functional } \\
\text { groups }\end{array}$ & Examples \\
\hline Oxidation & $\begin{array}{l}\text { Concentration of drug, } \\
\text { temperature, catalysts, } \\
\text { solvents, light and } \\
\text { excipients }\end{array}$ & $\begin{array}{l}\text { Aldehydes, alcohols, } \\
\text { phenols, alkaloids, } \\
\text { unsaturated alkyl chains, } \\
\text { carboxylic acids }\end{array}$ & $\begin{array}{l}\text { Paracetamol, progesterone, testosterone, quinine, } \\
\text { oils (unsaturated fats) such as soybean and corn oil, } \\
\text { essential fats, atorvastatin, atenolol }\end{array}$ \\
\hline $\begin{array}{l}\text { (O2 } \\
\text { dependent) }\end{array}$ & & 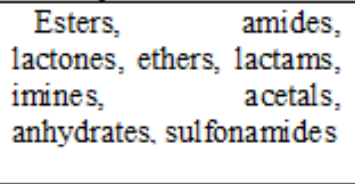 & $\begin{array}{l}\text { Aspirin, vigabatrin, norfloxacin, omeprazole, } \\
\text { simvastatin (statins), baclofen, diphenoxylate, } \\
\text { methylphenidate, lignocaine, sildenafil, penicillins, } \\
\text { cephalosporins, diazepam. digoxin, heparin. } \\
\text { captopril, hydrocortisone }\end{array}$ \\
\hline
\end{tabular}

*Drug molecules with more than one functional group can be more easily degraded. In fact, many drugs contain more than one functional group, being susceptible to both oxidation and hydrolysis, e.g. atenolol (contains amide and alcohol groups)

Figure 1. EXHIBIT 1

Exhibit 2. Why Is Off-Label Use of Drugs in Children Still a Problem? [13]

- Lack of specifications required for component development by compounding pharmacies.

- No onsite testing of active ingredients and excipients for purity, potency, content and stability.

- No onsite specifications or testing of product containers and closures.

- Site-to-site variations in compounding procedures, equipment, and the degree of product handling/manipulation.

- Lack of environmental control, which might lead to unintentional contamination and generation of degradation products due to inconsistent exposure to light, temperature and processing controls.

- Lack of testing of finished products for purity, potency, content or stability.

- Stability data for establishing expiry dates of compounded products are derived from published data, where preparation methods likely vary from local methods, or are simply default expiry periods defin ed by regional pharmacy regulations and "best practices".

- Published preparation methods provide only a portion of the information needed to consistently preparc a stable potent final product.

- Limited options available to mask bad-tasting active ingredients.

- The dose administration technologies used such as droppers, syringes, scoops, spoons, etc., vary between siles and between prescription fills.

- Weak regulatory oversight.

Figure 2. EXHIBIT 2.

equipment are readily available. Chemical and supply companies have increased in size and number in recent years and many provide information on compounding, incompatibilities, and stability. Specialty compounding organizations have developed over recent years and generally provide fullline services and products to the compounding pharmacist. Many national organizations provide continuing professional education programs in both non-sterile and sterile compounding. These entities provide services to compounding pharmacists ranging from selling only compounding aids to providing only chemicals. Others offer additional services to include formulas as well as consulting expertise by telephone or via the internet. This service can assist in the process of compounding a particular product that may be difficult.
Training and Experience: Pharmacists involved in upgrading and increasing the traditional aspects of extemporaneous compounding need to keep current with all the new tools of their trade, retrieve the old from storage, and put in a bit of practice using their scientific background and their art before they will be comfortable in exhibiting their skills. The pharmacist must ensure that the staff, facilities and systems in place are capable on a day-to-day basis of providing an adequate quality service able to meet the needs of patients. There should be an adequate number of competent personnel at all times. All staff involved in extemporaneous preparation must receive training Appropriate to their role. Typically, this will: (a) provide them with knowledge in good extemporaneous preparation practice, local practices including health and safety, formulation, expiry periods and quality assurance appropriate to the level of involvement, 
Exhibit 3. MHRA (2007) in the context of extemporaneous preparation [14]

- Extemporaneously prepared medicines should be formulated and prepared in compliance with current legal requirements and standards.

- Preparation and quality control arrangements are to be documented and in compliance with current GPP requirements.

- All products prepared should be quality suitable for their intended use

- Products are released for patient use only by a pharmacist.

- Documentation and records should comply recommendations in this guidance document.

Figure 3. EXHIBIT .3

assessment of risk and medication error potential, the pharmacy, its products and services provided (b) demonstrate competency in the necessary extemporaneous preparation skills and pharmaceutical calculations and dilutions. During training, staff must be carefully supervised and checked and trainees must understand the limits of their responsibilities. When considering providing additional services of compounding in an institution, pharmacists should not expect that this will change a great deal of their practice in time consumed for compounding. A written training program should be available and completion of training should be documented. This applies to all personnel working in the preparation area, including those not directly involved in preparation processes (e.g. cleaning staff). [14] .

Equipment: The equipment needed will be determined by the type and extent of the services one chooses to provide. Many pharmacies already have clean air environments (e.g., laminar air flow hoods, isolation barrier systems) where aseptic compounding of sterile solutions is performed. These same units can be used to compound other sterile preparations such as eye drops. A balance, preferably electronic, is essential. Ointment slabs (i.e., pill tiles), along with spatulas of different types and materials, should be purchased. A few mortars and pestles (i.e., glass, ceramic, plastic) and some glassware should be secured. A policy for the segregation of specific equipment for certain product types, including cytotoxic drugs and external products, should be available; this should take into account local COSHH assessments. Equipment should be calibrated and validated to demonstrate satisfactory performance. Measures should be calibrated for specific volumes and should have levelling lines. Glassware should be inspected regularly, before and after each use, for chips and cracks, and replaced as necessary. Consideration should be given to the merits of other materials. Stainless steel of a suitable grade is a good material but care must be taken with some agents as it is susceptible to oxidation. Plastics are susceptible to surface scratches and stains. The accuracy of balances should be checked on a regular basis and records of this process maintained. It may not be necessary to buy a roomful of equipment, but one should purchase what is needed to start the service and should build on it as the service grows and expands to different arenas. Much of the equipment used today in compounding has changed. Today, electronic balances are used more often than torsion balances; micropipets are commonplace; and ultra-freezers are sometimes required in addition to standard refrigerator freezers. This area is constantly changing and the compounding pharmacist should be aware of the available technology to prepare accurate and effective prescriptions. Becoming acquainted with the local representative for a laboratory supply company is helpful [2, 6, 14-19] .

Environment: A separate area for traditional compounding is recommended, rather than simply cleaning off a small area of the dispensing counter. The compounding pharmacist needs a clean, neat, well-lit and quiet working area. If aseptic compounding is considered, a clean air environment (e.g., laminar air flow hood, isolation barrier system) should be used. The actual facility to be used depends on the level and volume of compounding to be done. Extemporaneous compounding takes place in community and hospital pharmacies. There are usually specialist compounding pharmacies in major towns and cities, but any pharmacy may undertake compounding as long as they have appropriate facilities according to state-based legislation (e.g. allocated clean bench, specific compounding equipment). There should be a dedicated facility/room for extemporaneous preparation. However, for pharmacies preparing small quantities of a limited range of products for immediate dispensing then a designated area or temporarily designated area is permissible. The area must be clearly demarcated during preparation. Measures must be taken to reduce any risk of cross- contamination. Only one product should be handled at a time. Premises and equipment should be easy to clean. Following maintenance or repair, thorough cleaning and, where appropriate, disinfection should take place. Environmental conditions, including temperature, humidity and lighting, must be adequate to permit safe and comfortable preparation of medicinal products to limit the degradation of ingredients. Facilities should be laid out in a way to provide adequate space for the activities undertaken. Work should flow logically between working areas without the risk of product cross- contamination. Complex compounding is performed in a pressurized clean room using a laminar flow cabinet, cytotoxic drug safety cabinet or an isolator. Many public and private hospitals maintain large aseptic compounding facilities to provide individualized dosing or commercially unavailable formulations. Areas and equipment 
should be cleaned effectively with a suitable detergent before and after use. Equipment should be rinsed with an appropriate grade of water after washing (potable or sterile water for irrigation/injection) and then dried. Critical surfaces should be effectively sanitized with a suitable agent such as $70 \%$ alcohol before use. Sinks should be available for washing up of equipment, with separate facilities for handwashing. Sinks should be a suitable distance away from the preparation areas to reduce the risk of microbial contamination from water splashes. Washing and cleaning activities should not themselves be a source of contamination. All equipment should be visibly clean prior to use. Ensure that all residues of cleaning agent have been removed Containers and lids should be checked to ensure that they are clean and dry before use. Adequate pest control measures should be taken. [6, 10, 19]

Formulas: Consistency of the compounded product is important. Formulas should be developed or obtained and tried to assure that each time an extemporaneous product is prepared, the methods used, ingredients added, and the order of steps is documented. This accomplishes three things. First, it provides the methodology for each person involved or requested to provide such service the information necessary to do so properly. Second, it provides consistency from batch to batch. Third, if the product does not turn out the way expected, a stepwise methodology exists for reviewing and determining what happened and if revisions and improvements are needed $[6,15]$.

Chemicals and Supplies: If one is going to prepare a topical product, a vehicle (e.g., cream, ointment, gel) and the active ingredients (e.g., either finely ground product from an available tablet or injection or pharmaceuticalgrade chemicals) would be required. One needs proper dispensing containers for the medication. In short, a relationship with providers that carry chemicals and supplies is important. Pharmacists have been using chemicals and other materials for prescription compounding throughout history. In the past, these chemicals and materials have been obtained from natural products, raw materials, and household ingredients. Today, compounding pharmacists use chemicals from various reliable commercial sources, depending on their availability $[9,15-18]$.

Documentation: These give processing, packaging and release instructions. For products regularly prepared, master documents which combine the specification and product specific instructions should be produced and independently checked. Alternatively, a simple final product specification may be produced in addition to the master document. Master documents should be clear and detailed and should have a standardized style within any one pharmacy. Simple stepwise instructions for preparation, along with any specific notes or cautions (e.g. COSHH requirements) should be included on the master document for each product. A record should be kept of the key stages of processing, packaging and release of products to provide an audit trail of the quality relevant facts of the history of an extemporaneous product during preparation. This is normally achieved by completion of an individual worksheet, usually by photocopying the master document. Worksheets will vary for each pharmacy but should include: the name and formula of the product, and the source of the formula; a unique identification number to enable traceability; the manufacturer, batch numbers of each starting material (or QC reference number); the date of preparation etc. Labels must comply with all statutory and professional requirements, and should include the following information: the name of the product, and where necessary the nature of the salt and any waters of crystallization; quantity and strength of active ingredients; the pharmaceutical form etc $[6,20]$.

\section{Types of Compounding}

Active pharmaceutical ingredients can be incorporated into a wide array of products including creams, eye drops, nasal sprays, oral dosage forms or intravenous infusions. In Australia, products may be classified into simple or complex compounding Table 1 . Simple compounding can be performed by any pharmacist and is a core competency of pharmacy training. Complex compounding requires additional training and evidence, as described by the Pharmacy Board of Australia's guidelines on compounding. Finland, Italy, and Scotland prepared mainly powder, while Belgium, Croatia, France, and Switzerland prepared mainly capsules. Practice in Germany, Spain, and Slovenia involved preparation of a less well-defined combination of liquid, powders, and capsules.

Ambulatory-Care Compounding: If individuals can walk, they are considered mobile or ambulatory (i.e., they are not bedridden). Consequently, most pharmacists are involved in ambulatory care, and most ambulatory patients are outpatients. Actually, the term can also be applied to home-care patients and even institutionalized patients who are mobile. One general characteristic of ambulatory patients is that they are generally responsible for obtaining their own medication, storing it, preparing it (if necessary), and taking it. It seems almost incongruous that in health care today as we become more aware that patients are individuals, respond as individuals, and must be treated as individuals that some health-care providers appear to be grouping patients into categories. They are grouped in categories for treatment, for reimbursement from a third party, or for determining levels of care in managed-care organizations and using fixed-dose products provided by pharmaceutical manufacturers that are available because the marketing demand is sufficiently high to justify their manufacture and production. Why should the availability or the lack of availability of a specific economically profitable commercially available product dictate the therapy of a patient? Pharmacists have an opportunity to extend their activities in patient care as the emphasis continues to shift from inpatient care to ambulatory care. Ambulatory care, however, is so diverse and involves so many disciplines that sometimes it is difficult to understand it; and, it changes rapidly. Also, ambulatory care could generally encourage a team approach to health improvement, prevention, health maintenance, risk assessment, early detection, management, curative therapy, and rehabilitation. Ambulatory care offers various opportunities for individualizing patient care through pharmaceutical compounding. In fact, it is the area where most 
Table 1. Classification of simple versus complex compounding [5]

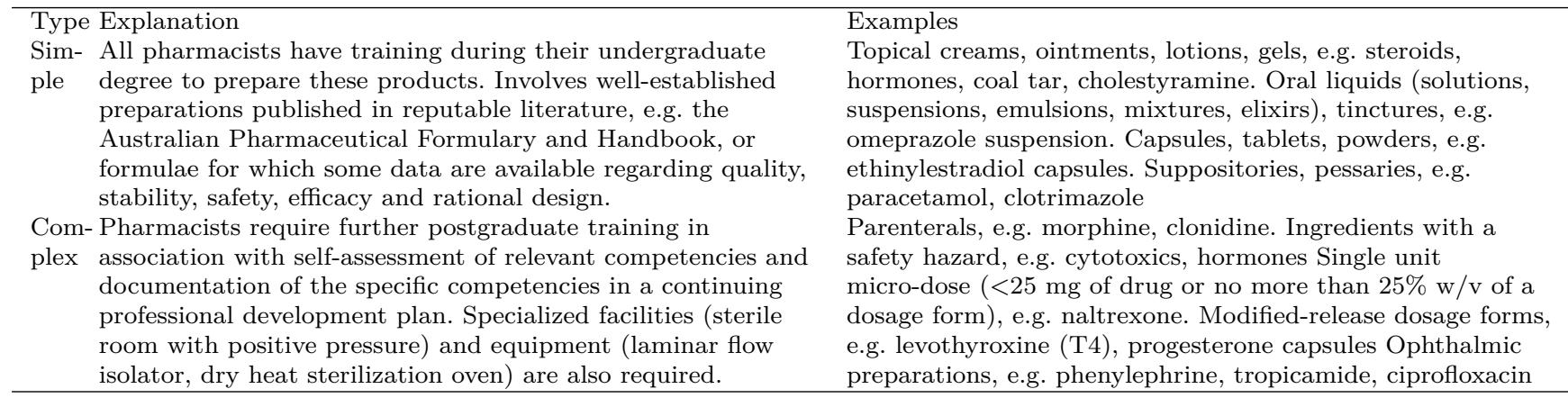

compounding pharmacists' practice. Pharmacists' roles in ambulatory care patients can include, among others

Dispensing

Compounding

Counseling

Minimizing medication errors

Compliance enhancement

Therapeutic drug monitoring

Minimizing expenditures

Most reimbursement for ambulatory patients comes from the dispensing or the compounding process. Little financial consideration is given to counseling, minimizing medication errors, compliance enhancement and therapeutic monitoring. However, these activities are important and should be performed. Because of the unique nature of compounded medications, counseling is an absolute must for these patients. From the above discussion of the activities of ambulatory care pharmacists, it should be evident that extemporaneous compounding can be vitally important in ambulatory patient care $[6,18-21]$

Hospital Pharmacy Compounding: The ever-present responsibility of the health-care industry is to provide the best available care for the patient, using the best means to do so, and providing that care in a conducive environment. This must be sufficiently economical to not put the institution in jeopardy of being unable to continue to provide the services to the community they serve. This requires cooperation on the part of the hospital administration, the medical staff, and the employees (nurses and pharmacists in particular as regards to medication usage) and must involve the patient. One of the effective means by which hospitals, and therefore hospital pharmacies, can meet these challenges is to consider expanding extemporaneous compounding services within the hospital pharmacy. Pharmaceutical care and pharmaceutical compounding can provide cost savings to the hospital while providing needed options to the physician through problem-solving approaches and stimulating the hospital pharmacist through new challenges that allow the expression of both their skills and their art [15, 22, 23]

Veterinary Compounding: The first symposium on veterinary compounding was a significant forum for discussion by experts and was a pivotal point in the history of veterinary compounding, occurring in September 1993. the meeting was important because it assembled an impressive group of experts on veterinary compounding, who then set about explaining and defining the roles of the veterinarian and the pharmacist [24] .

Nuclear Pharmacy Compounding - Nuclear pharmacy is a specialty practice of pharmacy that has been defined as a patient-oriented service that embodies the scientific knowledge and professional judgment required for improving and promoting health through assurance of the safe and efficacious use of radioactive drugs for diagnosis and therapy. Radioactive drugs, commonly referred to as radiopharmaceuticals, are a special class of drugs that are regulated by the FDA. They are unique in that they contain an unstable nuclide (radioactive nuclide) as a part of the compound designed to localize in an organ or tissue. Since radiopharmaceuticals are radioactive, the Nuclear Regulatory Commission or a similar state agency is involved in regulatory matters relevant to radiopharmaceuticals [25-27]

\section{Risk Management}

Legal Background: Pharmacists must comply with all legislation relevant to the practice of pharmacy in the jurisdiction where the practice occurs. Failure to practice in accordance with these requirements may give rise to action by one or more responsible authorities. These matters may then be referred to the Board for appropriate action under the National Law or law of a co-regulatory jurisdiction. Noncompliance with these guidelines and the practice standards and guidelines relevant to compounding may also be notified directly to the Board for appropriate action under the National Law or law of a co-regulatory jurisdiction. Noncompliance may be reported by an individual lodging a notification form, or through other means such as notification of outcomes of audits carried out by a state/territory pharmacy premises regulatory authority or responsible body [5]

\section{Alternatives to extemporaneous preparation}

Therapeutic substitution: The use of a licensed medicine from the same therapeutic classification should be considered and may provide a better clinical option than the use of an extemporaneously prepared medicine which has limited data to support its formulation and stability. The use of a less potent steroid rather than diluting a potent agent is an example where a therapeutic alternative may eliminate the need for an extemporaneous preparation. 


\begin{tabular}{|c|c|}
\hline \multicolumn{2}{|c|}{ Exhibit 4. Examples of Pre-1938 Drugs That Remained on the Market as Unapproved Drugs [15] } \\
\hline $\begin{array}{l}\text { Acetaminophen, codeine phosphate, and caffeine capsules and } \\
\text { tablets }\end{array}$ & Morphine sulfate oral solution and tablets \\
\hline Amobarbital sodium capsules & Nitrogycerin sublingual tablets \\
\hline Amyl nitrate inhalant & Opium tincture \\
\hline Chloral hydrate capsules, syrup, and suppositories & Oxycodone tablets \\
\hline Codeine phosphate injection, oral solution, and tablets & Oxycodone hydrochloride oral solution \\
\hline Codeine sulfate tablets & Paregoric \\
\hline Colchicine injection and tablets & Phenazopyridine hydrochloride tablets \\
\hline Digitoxin tablets & Phenobarbital capsules, elixir, and tablets \\
\hline Digoxin elixir and tablets & Phenobarbital sodium injection \\
\hline Ephedrine sulfate capsules and injection & Pilocarpine hydrochloride ophthalmic solution \\
\hline Ergonovine maleate injection and tablets & $\begin{array}{l}\text { Potassium bicarbonate effervescent tablets for oral } \\
\text { solution }\end{array}$ \\
\hline Ergotamine tartrate tablets & Potassium chloride oral solution \\
\hline Hydrocodone bitartrate tablets & Potassium gluconate elixir and tablets \\
\hline Hydrocodone bitartrate, aspirin, and caffeine tablets & Potassium iodide oral solution \\
\hline Hydromorphone hydrochloride suppositories & Salsalate capsules \\
\hline Levothyroxine sodium for injection & $\begin{array}{l}\text { Sodium fluoride oral solution and tablets } \\
\text { Thyroid tablets }\end{array}$ \\
\hline
\end{tabular}

Figure 4. EXHIBIT 4.

There are great opportunities for oncology pharmacists, as well as community pharmacists, as a resource for educating and monitoring patients receiving oral chemotherapy to ensure dosing accuracy, safe administration, and proper disposal of hazardous drugs. Oncology pharmacists face a constant challenge with patients who cannot swallow oral anticancer drugs, making extemporaneous oral liquid preparation a requirement. Improper extemporaneous preparation of these agents, especially with the traditional chemotherapy with a narrow therapeutic index, may increase the risk of over- or under-dosing [28] .

Use of an imported product: The preparation selected should be licensed for use in a country with equivalent or similar licensing arrangements and regulatory standards to the UK (e.g. EU, Canada, Australia). This will provide the requesting pharmacist with assurance that the quality, safety and efficacy of the medicine have been reviewed by a competent regulatory authority. Although clinicians do not doubt the efficacy and utility of captopril in managing children with heart failure, concerns exist about optimal dosing schedules and toxicity. This formulation is licensed in the Bristol-Myers Squibb, Australia, but was not available in the UK [29] .

Use of a 'Special' manufactured in a MHRA licensed unit: The benefit of purchasing a 'Special' is that the product should be made to a validated formula with supporting stability data in accordance with the principles of GMP. Licensed 'Specials' units are regularly inspected by the MHRA to ensure these principles are upheld. However, the purchasing pharmacist will still need to review the supporting documentation (e.g. specification, Certificate of Analysis/Conformity, BSE/TSE statement) to assess whether the product is of appropriate quality. Information on Specials manufacturers is available in the BNF. 
Specials may be used to meet a variety of patient needs, for example, for babies, children and older people, and in specific areas such as dermatology and palliative care [30] .

Use of soluble or dispersible tablets: This practice presents fewer health and safety risks than crushing tablets, which can expose the carer to potentially harmful dusts via inhalation. When dispersing tablets, the dose should be prepared and administered immediately, as stability cannot be guaranteed. It should be noted that slow or modified release preparations should not be used in this manner. If the active is not soluble, it can lead to inaccuracy of dosing through a lack of dose uniformity and reproducibility. This is a major consideration when no suspending agents are used, especially when the person administering the dose is inexperienced and the dose is small [31] . Milk has been explored as a vehicle in liquid formulations showing potential for solubilizing drugs while maintaining the stability of the emulsified vehicle [32] .

Cutting tablets: Tablets cannot be cut with great accuracy of dose and research suggests that the variability may range from $50 \%$ to $150 \%$ of the desired dose even when using commercially available tablet cutters.

Use of a preparation intended for a different route: The use of a suitable preparation intended for a different route of administration can sometimes be a practical alternative; for example, the use of an injection solution orally, or an oral solution rectally. However, this practice has its own inherent risks and the pharmacist should ensure that the presentation used will be absorbed by this route and that it will be tolerated by the patient. When using an injection by the oral route, consideration should be given to the possibility of rapid absorption and elevated peak levels, the potential for rapid drug degradation due to exposure to gastric acid and problems with first-pass metabolism. The $\mathrm{pH}$ of an injection should also be considered, as extremes of pH can adversely affect the gastric mucosa [20] .

Risks associated with extemporaneous preparation

Formulation failure: An insoluble drug suspended in a suitable vehicle may be less susceptible to drug degradation, but may settle out of the suspension over time, leading to sedimentation and caking. In this state, there will be a higher concentration of drug at the bottom of the bottle than at the top. In 2006, the FDA conducted a limited survey of compounded drugs. Of 36 samples tested by the FDA, 12 failed at least one quality test, for a failure rate of $33 \%$. Further, oral hormone dosage forms containing multiple active ingredients showed poor content uniformity, with random variation in all three active ingredients from capsule to capsule $[8,20]$.

Microbial contamination: The choice of preservative for a formulation needs to take into account a number of factors including $\mathrm{pH}$, physical compatibility and the intended patient group. Unpreserved preparations should be stored in a refrigerator and assigned a short shelf-life to limit microbial growth. A maximum shelf-life of 7 days at $2-8^{0} \mathrm{C}$ should be assigned to unpreserved oral liquid preparations unless sufficient validation work has been carried out to support an extended shelf-life. Essential macronutrients and micronutrients infused via PN solutions are an essential part of the care of infants and children who cannot or should not be fed enterally. Infants and children require a greater amount of fat in their diet than do adults to support growth and development. The use of IVFE provides a concentrated source of calories and prevents the development of essential fatty acid deficiency in neonates fed exclusively via the parenteral route. The AAP recommends IVFE doses of up to 3 $\mathrm{g} / \mathrm{kg} /$ day (approximately $30 \mathrm{kcal} / \mathrm{kg} /$ day) for use in infants. A variety of microorganisms have been found in residual IVFE after completion of the infusion [33] . Microorganisms could potentially be introduced during reformulation of non-sterile products. For example, if Candida albicans is inadvertently introduced into freshly prepared multi-dose citric acid solutions that are to be used orally for cough reflex testing, immunocompromised individuals could become infected. Regular monitoring of the environment, equipment and procedures is essential to ensure quality and sterility is maintained. The consequences of failure can be catastrophic, for example methylprednisolone injections from a single compounding pharmacy in the USA resulted in 137 cases of Aspergillus fumigatus meningitis and 12 deaths [2]

Calculation errors: Common calculation errors associated with extemporaneous preparation include errors when converting units from one to another (e.g. milligrams to micrograms, conversions from weight in volume to millimoles). Dangers of potent ingredients and calculation errors, particularly where the strength of one or more ingredient is stated in a historical or non-standard fashion. Similar reports have originated from the US, with the death of a child from a super-potent imipramine liquid, and a five-year-old child who received a 1,000-fold overdose of clonidine. Problems can also arise when doses can be prescribed as free base or salt, leading to potential calculation errors when making and administering preparations (e.g. two-fold errors if caffeine citrate is confused with caffeine base). Some of these errors have been attributed to inconsistent labelling approaches, for example, strengths expressed per millilitre or per $5 \mathrm{ml}$ spoonful. Clinical pharmacists in pediatrics and neonatology help in dose calculation and dosage form modification. The increased need for calculations and dilutions of pediatric medicines, and a requirement to adjust dose of an individual patient based on age, gestational age, weight and surface area, provide a greater opportunity for a clinical pharmacist in medication management process. They can help to prepare pediatric/neonatal formulary [34-41] .

Starting materials: Care should also be taken with the use of cariogenic sugars (e.g. sucrose) in pediatric formulations as it has been associated with dental cavities. It is therefore important to list all such excipients on the product label so that end-users are made aware of their presence in the formulation. In a 2004 published analysis sponsored by STD Pharmaceuticals, all samples purchased from three compounding pharmacies failed content testing for a $3 \%$ sodium tetradecyl sulfate solution for injection (range 2.593.39). Significant concentrations of the contaminant carbitol were found to be present in samples from all three sources 
(0.33-4.18), suggesting possible use of a non-pharmaceutical grade chemical [34] .

Patient acceptability issues: Consideration should be given to the palatability and presentation of oral liquid medicines as there is a good argument that taste is crucial to achieving good compliance in children, especially for the treatment of longstanding conditions such as in cardiology. Extemporaneously prepared oral drug formulations can also be plagued by poor palatability characteristics which can compromise patient adherence. This lack of child-friendly formulations affects $40 \%$ of the global population, subjecting pediatric patients to avoidable adverse drug events, reduced compliance with medication regimens, limited access to new medications, and prolonged treatable illnesses. The pressing need for child-friendly, palatable medications suitable for administration to both infants and young children has been stressed (through regulatory guidance) by both the European Medicines Agency and the US FDA. Prednisone is a bitter-tasting corticosteroid used for its anti-inflammatory and immunosuppressant effects to treat a wide variety of conditions in both adults and children. For pediatric patients, an alternative form of administration can be produced by crushing solid oral prednisone formulations in order to avoid swallowing difficulties and/or achieve weight-based dosing. This approach, however, creates extremely poor taste and mouth feel properties which are objectionable to pediatric patients. While these limitations can be somewhat mitigated by the use of proprietary oral liquid formulations of prednisone, such products still retain their profoundly bitter taste characteristics. It is estimated that approximately half of children refuse to take even a liquid form of prednisone, with the large majority of those reporting bad taste as the single major reason for non-compliance. Efforts to mask flavors using sweetening agents, coatings, agglomeration, or microencapsulation often result in poorly-controlled, heterogeneous particle size distributions that result in a gritty or granular mouth feel and can provide ineffective taste masking, characteristics that may also compromise patient acceptance. The design of an ideal paediatric formulation needs to consider the following factors: (i) producing minimal impact on the lifestyle of the child, manifesting as the lowest dosage frequency and a palatable product, (ii) provision of individualized dosing or dose banding appropriate for effective therapy, (iii) sufficient bioavailability, (iv) non-toxic excipients in the formulation, (v) convenient and reliable administration and (vi) robust production process at minimal cost [20, 36-39] .

Health and safety risks: When handling hazardous products, units should be equipped with suitable containment devices and systems should be put in place to eliminate the risk of cross-contamination.

Therapeutic risks and clinical consequences: Any inaccuracy of dosing associated with medicines that have a narrow therapeutic index can lead to significant morbidity, whether due to under-dosing leading to treatment failure or overdosing leading to toxicity. By contrast, any inaccuracy of dosing associated with drugs with a wide therapeutic index may have little or no impact on the therapy.
Associated clinical risk factors: The majority of patients receiving extemporaneously prepared products, in particular oral liquid medicines, tend to be from vulnerable patient groups (e.g. neonates, children, stroke victims) who are either unaware of ill-effects associated with their treatment or who cannot communicate with their clinician. Coupled with this, extemporaneous preparations may not be routinely identified as high-risk therapies by pharmacists and therefore such treatments are not commonly given the level of scrutiny and close monitoring they require. Therefore, when embarking on the use of an extemporaneously prepared product, the pharmacist should ensure that systems are in place to monitor the effectiveness of the therapy [20] .

\section{Risk Management}

The European Pharmacopoeia monograph on Pharmaceutical Preparations specifies the necessity for tests to be applied to particular dosage forms, as described in their respective dosage form monographs, which are also applicable in the case of extemporaneously prepared products. The requirements on testing in the European Pharmacopeia monograph deal with the appearance of products, identity and purity tests, uniformity and reference standards. The extent of quality control applied to a product should be proportionate with the level of risk the finished product could pose to the patient and should be judged on a case by case basis. Consideration should be given to the individual patient's age and condition, whether the product will be ingested or used topically, as well as the potency of, and risk posed by, the active substances and/or excipients. Where it is not practical to carry out this testing (e.g. due to the batch size, urgency, etc.), other suitable methods should be implemented to ensure that the appropriate quality is achieved, in accordance with the risk assessment carried out. At a minimum, the starting materials and finished product should be examined visually before supply to a patient [40]

\section{Role of the Pharmacists}

Compounding preparations extemporaneously on lawful prescription for individual patients is within the pharmacist's purview. Pharmacists and pharmacies are licensed and inspected by the states; if a state inspection uncovers "manufacturing under the guise of compounding," it refers the matter to the FDA, which can apply cGMP expectations to the pharmacist/pharmacy. When a pharmacist engages in large-scale production of preparations without prescription or evidence of pending refills, trending, etc, the practice can be regarded as manufacturing; cGMP compliance will be expected, including an approved NDA or ANDA. Today, however, compounding pharmacies have begun to flourish and are once again becoming a vital component of pharmacy practice. Many health care professionals recognize that, for some specific patient populations, there is a growing need to compound certain medications because they are not available through conventional manufacturing methods. Whereas the services provided by compounding pharmacies give many patients options with regard to drug therapy, compounding pharmacists must be properly trained to specialize in this aspect of pharmacy practice and must have 
the appropriate resources and references to compound quality medications. Pharmacists are responsible for ensuring that extemporaneous preparations are compounded according to compounding guidelines and standards with respect to purity, quality, stability, packing, record keeping, and other appropriate pharmacy practices. Compounding pharmacists must work closely with the prescribing physician to ensure that patient goals are met. A positive therapeutic outcome for the patient is the fundamental goal for all health care professionals, and, with that goal in mind, the services provided by compounding pharmacies can continue to make pharmacists an indispensable part of the health care team $[35,41]$.

\section{Epilogue}

Pharmacy compounding provides pharmacists with a unique opportunity to practice their time-honored profession. It is becoming an even more important part of pharmacy practice in the future, including those involved in community and hospital care, nursing care, home care, veterinary and other specialty practices. It is a practice where the clinical expertise can be merged with the scientific expertise of pharmacists to make a visible pharmaceutical care. Pharmacists are to be encouraged more in compounding but should be aware of the core of formulating a specific drug product for a specific patient. This is important in providing pharmaceutical care.

\section{REFERENCES}

[1] Taylor S, Hays C, Glass B. 2018 PJMH, editor. Compounding medications in a rural setting: an interprofessional perspective; 2018.

[2] JR SKF. Extemporaneously compounded medicines. Aust Prescr; $1(5-8)$.

[3] RP MCL, CM C, DJ C, RD C, B C, P P. Extemporaneous preparation strategy for early phase clinical studies. Int. vol. 549; 2018. 25. PubMed PMID: 30055303.

[4] Brown G. The Value of Drug Stability Studies and Their Publication. Can J Hosp Pharm;3(161-162).

[5] Pharmacy Board of Australia. Codes, guidelines and policies. Guidelines on compounding medicines. 2015;. Available from: http://www.pharmacyboard.gov.au/CodesGuidelines.aspx.

[6] L V. Chapter 105. Extemporaneous Prescription Compounding. In: Remington: The Science and Practice of Pharmacy by David B. Troy: Paul; 2006. Beringer, published by Lippincott Williams \& Wilkins.

[7] Kei C, Tuleu C, Yeung V. Paediatric Drug Handling. Long: Ian; 2007.

[8] H W. Pharmacy compounding primer for physicians: prescriber beware. Drugs;16(2043-50).

[9] A V. The Art, Science and Technology of Pharmaceutical Compounding Fifth Edition APhA Wshington. D.C;

[10] Jaksic ML, Malenovic A, Reichl S, Hoffmann C, MüllerGoymann C, Rolf. Daniels and Snezana Savic, Compounding of a topical drug with prospective natural surfactantstabilized pharmaceutical bases: Physicochemical and in vitro/in vivo characterization - A ketoprofen case study. European Journal of Pharmaceutics and Biopharmaceutics. 2012;80(1). 10] Ivana.

[11] J JM, J C, M R. Potential risks of pharmacy compounding. Drugs R D;1(1-8).
[12] Alarie H, Friciu MM, Leclair G. Stability of Dapsone in Extemporaneously Compounded Oral Suspensions. Can J Hosp Pharm;2(152-154).

[13] V MRB, R L. Cies JJ, Cernak I, Ii RHP. An Algorithm to Identify Compounded Non-Sterile Products that Can Be Formulated on a Commercial Scale or Imported to Promote Safer Medication Use in Children. Pharmacy (Basel). 2015 11;4(284-294):11.

[14] Handbook of Extemporaneous Preparation: A Guide to Pharmaceutical Compounding. In: Rowe P, editor. the Pharmaceutical Press. London, Chicago;. p. 29. Issue 10 (Oct 2010.

[15] L V. Chapter 1. Guidelines for Compounding Practices. In: Loyd. Edition, publisher American Pharmacists Association; 2017.

[16] Extemporaneous formulations reference guide The Pharmaceutical Journal24; 2016. By Laurence A. Goldberg.

[17] Medicines, Ethics, and Practice: A Guide for Pharmacists, Volume 29 by. Royal Pharmaceutical Society of Great Britain. 2005;

[18] McHugh MB. Chapter 15. Non-sterile compounding and Repackaging. In: Mary B. McHugh. Workbook for the Manual for Pharmacy Technicians, published by ASHP; 2013.

[19] Parts 1-2; Volumes 2-3 by United States Pharmacopeial Convention. vol. 1; 2006

[20] Handbook of Extemporaneous Preparation: A Guide to Pharmaceutical Compounding by. Pharmaceutical Press; 2010 .

[21] The Pharmacy Technician by Jahangir Moini, published by Cengage Learning; 2010.

[22] Sundberg JA. Extemporaneous Compounding in the Hospital Pharmacy. International Journal. 1997;Available from: http://www.ijpc.com/Products/ProductAddToCart.cfm.

[23] Thompson A. Recent FDA compounding guidances to pharmacies may not apply everywhere. American Journal of Health-System Pharmacy, Volume. 2018 3;75(5):258. Available from: https://doi.org/10.2146/news180015.

[24] D. Veterinary Compounding: Regulation, Challenges, and Resources Pharmaceutics; 2017. 24] Gigi.

[25] JC BLM, EM S. Section: Nuclear Pharmacy. Career Pathway Evaluation Program 2007 Pharmacist Profile Survey; 2007.

[26] a;. Available from: http://www.ncbop.org/LawsRules/rules. 2700.pdf.

[27] APhA Home. Nuclear Pharmacy Practice;. Available from: https://pharmacist.com/nuclear-pharmacy-practice.

[28] Lam MS. Extemporaneous compounding of oral liquid dosage formulations and alternative drug delivery methods for anticancer drugs. Pharmacotherapy; 2011. Review. PubMed PMID: 21275495.

[29] H TM, F B, N S, HC P. Variations in captopril formulations used to treat children with heart failure;. a survey in the United kingdom. Arch Dis Child. 2007;92(5):409-11.

[30] Special feature. Why specials manufacturing units are needed now as much as they ever were. The Pharmaceutical Journal (Vol. 2005 12;275:143-144.

[31] Patel VP, Desai TR and others. EXTEMPORANEOUS DOSAGE FORM FOR ORAL LIQUIDS. Pharmacophore;2011(2):86-103.

[32] Lopez FL, Ernest TB, Tuleu C, Gul MO. Formulation approaches to pediatric oral drug delivery: benefits and limitations of current platforms. Expert Opin Drug Deliv;11(172740)

[33] Crill M, Hak EB, Robinson LA, Helms RA. Evaluation of microbial contamination associated with different preparation methods for neonatal intravenous fat emulsion infu- 
sion. American Journal of Health-System Pharmacy, Volume. 2010 6;67(11):914-918. Available from: https://doi. org/10.2146/ajhp090199.

[34] Goldman MP. Sodium tetradecyl sulfate for sclerotherapy treatment of veins: is compounding pharmacy solution safe? Dermatol Surg;12(1454-6).

[35] A M. Your Compounding Questions Answered. Pharmacy Times $^{\circledR} .2013$ 9;

[36] S DN, C S. Palatability of a novel oral formulation of prednisone in healthy young adults. J Pharm Pharmacol;4(489496).

[37] Gee SC, Hagemann TM. Palatability of liquid antiinfectives: clinician and student perceptions and practice outcomes. J Pediatr Pharmacol Ther;4(216-23).

[38] P SH, C C, J H, H B. SPaeDD-UK project. Evaluation of patient-reported outcome measurements as a reliable tool to measure acceptability of the taste of paediatric medicines in an inpatient paediatric population. BMJ Open. 2018 $7 ; 2018(8): 11$.

[39] A SGJ, M V, C GM, M S. Fariña JB. Effectiveness of Antimicrobial Preservation of Extemporaneous Diluted Simple Syrup Vehicles for Pediatrics. J Pediatr Pharmacol Ther;5(405-409).

[40] Guidance for Pharmacists on Extemporaneous Dispensing Pharmaceutical Society of Ireland. Version. 2015 6;1.

[41] Terrie YC. Pharmacy Compounding Is Flourishing Once Again. Pharmacy Times ${ }^{\circledR}$. 2005 11;

\section{AUTHOR BIOGRAPHY}

AK Mohiuddin Assistant Professor, Department of Pharmacy, World University of Bangladesh, Bangladesh 